\title{
Once-daily fosamprenavir with ritonavir in the treatment of HIV infection in therapy-naïve patients
}

\author{
Leo Flamholc' \\ Magnus Gisslén² \\ 'Department of Infectious Diseases, \\ Malmö University Hospital, Malmö, \\ Sweden; ${ }^{2}$ Sahlgrenska University \\ Hospital/Östra, Gothenburg, Sweden
}

\begin{abstract}
Treatment options for HIV patients have dramatically improved since the introduction of efficacious antiretroviral combination therapy more than a decade ago. Treatment regimens have been simplified with fewer pills and fewer daily dosages. Fosamprenavir is a protease inhibitor with a rather long half-life which makes it a candidate for once-daily use. Once-daily dosage of ritonavir-boosted fosamprenavir is approved in the US, but not in Europe, for treatment in patients without prior antiretroviral treatment. Here we review the background and rationale for once-daily dosage of ritonavir-boosted fosamprenavir. The rather limited studies that have been published so far indicate that fosamprenavir $1400 \mathrm{mg}$ may be used once daily boosted with ritonavir. The optimal ritonavir dose to be given together with fosamprenavir is still to be defined, though available results indicate that a dose of $100 \mathrm{mg}$ may be adequate provided that no protease inhibitor resistance is present.
\end{abstract}

Keywords: HIV, fosamprenavir, ritonavir

\section{Introduction}

Antiretroviral drug combinations including two nucleoside reverse transcriptase inhibitors (NRTI) and a protease inhibitor (PI) were introduced in the mid 1990s. These drug combinations provided potent options for HIV-infected patients to durably suppress HIV to undetectable levels and dramatically reduced morbidity and mortality in HIV infection. Complicated dosing regimens and food restrictions had initially a negative impact on adherence and quality of life. A major improvement was achieved with the principle of boosting by administrating PIs together with a low dose of ritonavir to improve pharmacokinetic properties, making more convenient dosing options possible and thus improving adherence and virological outcome. At the present time eight different PIs are licensed in the US and Europe. With the exception of nelfinavir all PIs (indinavir, saquinavir, lopinavir, fosamprenavir, atazanavir, tipranavir, and darunavir) can or should be used together with low dose ritonavir.

\section{Once-daily dosing of protease inhibitors}

Only atazanavir is exclusively licensed for once-daily use. However, a number of studies have been performed in which other PIs have been evaluated in once-daily regimens. In a recent study lopinavir/r was given once or twice daily together with co-formulated tenofovir/emtricitabine in treatment-naïve patients. After 48 weeks lopinavir/r given once daily was shown to be non-inferior to twice-daily therapy (Gathe et al 2008).

Also darunavir/r given once daily to treatment-naïve patients has been studied. In the Artemis study it was compared to lopinavir/r (given once or twice daily) and was shown to be non-inferior. The darunavir/r dose was $800 \mathrm{mg}$ together with $100 \mathrm{mg}$ ritonavir. 
Also in this study the nucleoside backbone was co-formulated tenofovir/emtricitabine (Ortiz et al 2008).

The Gemini Study was a 48-week trial that randomized 337 treatment-naïve patients to either saquinavir/r or lopinavir/r, together with two NRTIs. The old capsule formulation of lopinavir/r was used twice daily and the saquinavir dose was $1000 \mathrm{mg}$ together with $100 \mathrm{mg}$ ritonavir. The NRTI backbone was tenofovir/emtricitabine. No statistically significant differences were found between the 2 regimens after 48 weeks treatment (Walmsley et al 2007).

In the US, once-daily use of ritonavir-boosted lopinavir and fosamprenavir are licensed and recommended as alternative PIs in patients without prior antiretroviral treatment (Panel on Antiretroviral Guidelines for Adults and Adolescents 2008). FDA has approved both 100 and $200 \mathrm{mg}$ of ritonavir once daily together with $1400 \mathrm{mg}$ fosamprenavir. In Europe neither lopinavir/r nor fosamprenavir/r are licensed for use once daily. Despite this, The European AIDS Clinical Society (EACS) guidelines 2007 recommend lopinavir/r, fosamprenavir/r, and saquinavir/r given once daily as alternatives for treatment-naïve patients in combination with two NRTIs (Clumeck et al 2008).

\section{Treatment with fosamprenavir/r}

The PI amprenavir was approved by the FDA 1999. Because amprenavir has low water solubility, a high rate of excipients to drug is required leading to large pills and a limited amount of drug in each pill, and a dose of 8 large pills twice daily. The use of amprenavir was limited by the large pill size and high pill burden. To overcome these obstacles the pro-drug fosamprenavir was developed. It is a phosphate ester pro-drug of amprenavir with improved water solubility, making it possible to make smaller pills with more active drug in each pill and allowing more convenient dosage. Fosamprenavir is metabolized to amprenavir in the epithelial cells of the intestine. The pro-drug itself is not substantially absorbed and the systemic exposure to fosamprenavir is low (Furfine et al 2004).

A 6-week, randomized, controlled, double-blind and crossover study in treatment-naïve HIV-positive patients showed that fosamprenavir delivered similar amprenavir concentrations compared to standard-dose amprenavir (Wood et al 2004).

Amprenavir has a terminal half-life of 8 hours (Sadler et al 1999) and it is metabolized principally by P450 CYP 3A4. Boosting with ritonavir increases $\mathrm{AUC}$ and $\mathrm{C}_{\min }$ of amprenavir using a non-compartmental pharmacokinetic method (Sadler et al 2001).
The NEAT study was a 48-week study in treatment-naïve patients randomized to either non-boosted fosamprenavir $1400 \mathrm{mg}$ bid or $1250 \mathrm{mg}$ nelfinavir bid with a backbone in both arms of abacavir/lamivudine. The randomization was 2:1 with 166 patients receiving fosamprenavir and 83 nelfinavir. Sixty-six percent of the patients on fosamprenavir versus $51 \%$ on nelfinavir had viral loads below 400 copies $/ \mathrm{mL}$. Diarrhea was significantly more common in the nelfinavir group. This was the only significant difference found in side effects (Rodriguez-French et al 2004).

Ritonavir-boosted fosamprenavir was compared to boosted lopinavir in the KLEAN study, a large non-inferiority study in treatment-naïve patients that enrolled 837 patients. Patients were randomized to either boosted lopinavir in standard dose twice daily or fosamprenavir $700 \mathrm{mg}$ twice daily together with $100 \mathrm{mg}$ ritonavir twice daily. All patients received background therapy of abacavir together with lamuvidine. After 48 weeks, non-inferiority was shown for fosamprenavir/r, 73\% of patients achieving viral load below 400 copies for fosamprenavir/r versus $71 \%$ for lopinavir/r. In summary there were no differences in efficacy, tolerability, or safety between the two treatments (Eron et al 2006). Similar results were reported in an open-label, observational study on 82 therapy-naïve HIV-infected subjects followed for 18 months (Calza et al 2008).

\section{Fosamprenavir administered once daily}

As stated above, because amprenavir has a rather long half-life once-daily therapy is feasible. The SOLO study compared once-daily fosamprenavir with $200 \mathrm{mg}$ ritonavir and nelfinavir given twice daily. Abacavir and lamuvidine were given twice daily to patients in both treatment arms. A total of 649 patients were enrolled. At 48 weeks $69 \%$ achieved viral load below 400 copies and $55 \%$ below 50 copies in the fosamprenavir $/ \mathrm{r}$ arm. The corresponding figures for nelfinavir were $68 \%$ and $53 \%$. Viral failure occurred in $17 \%$ of nelfinavir patients versus $7 \%$ in patients receiving fosamprenavir/r. Diarrhea was significantly more common in patients receiving nelfinavir. Total cholesterol increased $38 \%$ and $37 \%$, respectively, in the two treatment groups. In summary the virological results were somewhat disappointing with $55 \%$ and $53 \%$ reaching viral load below 50 copies. No genotypic resistance mutations to amprenavir were however detected (Gathe et al 2004).

Even at low doses, ritonavir causes lipid elevations and gastrointestinal side effects (Cooper et al 2003). To explore the possibilities of further reducing the dose of ritonavir 
in once-daily fosamprenavir therapy, a pharmacokinetic crossover study in 36 healthy volunteers was performed (Ruane et al 2007). Fosamprenavir was given once daily at $1400 \mathrm{mg}$ together with either 100 or $200 \mathrm{mg}$ of ritonavir during 14 days. After a washout period of 21 to 28 days medication was resumed for another 14 days with the alternate ritonavir dose. Steady state plasma amprenavir concentrations were measured at day 14 of each medication cycle. Equivalence between regimens for $\mathrm{C}_{\max }$ and $\mathrm{AUC}$ of the two ritonavir doses were shown. $\mathrm{C}_{\min }$ was $38 \%$ lower for those receiving $100 \mathrm{mg}$ of ritonavir. The $\mathrm{C}_{\min }$ was, however, 2.5 times higher than the $\mathrm{C}_{\text {min }}$ that is achieved with unboosted fosamprenavir $1400 \mathrm{mg}$ twice daily, which is an approved dose in the US. Based on these observations a treatment study was initiated comparing once-daily fosamprenavir in treatment-naïve patients with either 100 or $200 \mathrm{mg}$ ritonavir during 96 weeks; 115 patients were included (Hicks et al 2007). Interim results after 48 weeks show that $79 \%$ of patients in the $100 \mathrm{mg}$ group compared to $63 \%$ in the $200 \mathrm{mg}$ group had viral loads less than 50 copies $/ \mathrm{mL}$. The virological efficacy was higher in the $100 \mathrm{mg}$ group but there were no tolerability or lipid advantages observed with the lower ritonavir dose. Both the improved virological response and the absence of tolerability and lipid advantages may be explained by differences in adherence. The adherence was estimated to be higher in the $100 \mathrm{mg}$ ritonavir arm. In another randomized open label trial in treatment-naïve patients once-daily fosamprenavir boosted with $100 \mathrm{mg}$ ritonavir was compared with boosted atazanavir in the ALERT study (Smith et al 2008). All patients were also treated with a backbone of tenofovir and emtricitabine; 106 patients were enrolled and 48 weeks results provided comparable results for virological outcome, CD4 increase, lipid changes, and adverse events. Stratification according to baseline viral load above or below 100000 copies $/ \mathrm{mL}$ did not change the comparison between the two arms. In the Context study treatment-experienced patients who had a treatment history of one or two protease inhibitors and viral load greater than 1000 were randomized to either fosamprenavir given once or twice daily or boosted lopinavir dosed once daily (DeJesus et al 2003). Patients randomized to fosamprenavir once daily received $200 \mathrm{mg}$ of ritonavir and for patients on a twice-daily regimen $100 \mathrm{mg}$ of ritonavir was given together with $700 \mathrm{mg}$ of fosamprenavir. Each regimen was given together with two nucleoside reverse transcriptase inhibitors selected according to resistance tests. After 24 weeks of treatment non-inferiority was demonstrated for both fosamprenavir arms compared to lopinavir according to an ITT analysis of time averaged change in vRNA from baseline (AAUCMB). A total of 320 patients were enrolled. In summary the results are too limited for any conclusions on the utility of fosamprenavir given once daily to treatmentexperienced patients, and the regimen is not recommended for these patients.

\section{Discussion}

Treatment options for HIV patients have dramatically improved since the introduction of efficacious antiretroviral combination therapy more than a decade ago. Treatment regimens have been simplified with fewer pills and fewer daily dosages. Fosamprenavir is a PI with a rather long half-life that makes it a candidate for once-daily use. The rather limited studies published so far indicate that fosamprenavir may be used once daily boosted with ritonavir. Once-daily fosamprenavir is already listed as an alternative treatment in antiretroviral-naïve patients according to the Panel on Antiretroviral Guidelines for Adults and Adolescents 2008 and in The European AIDS Clinical Society (EACS) guidelines. The optimal ritonavir dose to be given together with fosamprenavir is, however, still to be defined though available results indicate that a dose of $100 \mathrm{mg}$ may be adequate, at least in the absence of PI resistance.

\section{Disclosures}

Neither author has conflicts of interest to disclose.

\section{References}

Calza L, Manfredi R, Pocaterra D, et al. 2008. Efficacy and tolerability of a fosamprenavir-ritonavir-based versus a lopinavir-ritonavir-based antiretroviral treatment in 82 therapy-nayive patients with HIV-1 infection. Int J STD AIDS, 19:541-4.

Clumeck N, Pozniak A, Raffi F. 2008. European AIDS Clinical Society (EACS) guidelines for the clinical management and treatment of HIV-infected adults. HIV Med, 9:65-71.

Cooper CL, Van Heeswijk RP, Gallicano K, et al. 2003. A review of low-dose ritonavir in protease inhibitor combination therapy. Clin Infect Dis, 36:1585-92.

DeJesus E, LaMarca A, Sension M, et al. 2003. The Context Study: Efficacy and safety of GW433908/RTV in PI-experienced Subjects with viroogical failure (24 weeks results). 10th Conference on Retroviruses and Opportunistic Infections. Boston, MA, USA.

Eron J Jr, Yeni P, Gathe J Jr, et al. 2006. The KLEAN study of fosamprenavirritonavir versus lopinavir-ritonavir, each in combination with abacavirlamivudine, for initial treatment of HIV infection over 48 weeks: a randomised non-inferiority trial. Lancet, 368:476-82.

Furfine ES, Baker CT, Hale MR, et al. 2004. Preclinical pharmacology and pharmacokinetics of GW433908, a water-soluble prodrug of the human immunodeficiency virus protease inhibitor amprenavir. Antimicrob Agents Chemother, 48:791-8.

Gathe JC Jr, Ive P, Wood R, et al. 2004. SOLO: 48-week efficacy and safety comparison of once-daily fosamprenavir/ritonavir versus twice-daily nelf inavir in naive HIV-1-infected patients. Aids, 18:1529-37. 
Gathe JDS, Loutfy B, Podzamczer M, et al. 2008. Study M05-730 primary efficacy results at week 48: Phase 3, randomized, open-label study of lopinavir/ritonavir tablets once daily vs twice daily, co-administered with tenofovir $\mathrm{df}+$ emtricitabine in ARV-naïve HIV-1-infected subjects. 15 th Conference on Retroviruses and Opportunistic Infections. Boston, MA, USA.

Hicks CD, Wohl E, Liao D, et al. 2007. Once-daily fosamprenavir (FPV) boosted with either $100 \mathrm{mg}$ or $200 \mathrm{mg}$ of ritonavir (r) and combined with abacavir (ABC)/lamivudine (3TC): 48-week safety and efficacy results from COL100758. 11th European AIDS Conference. Madrid, Spain.

Ortiz R, Dejesus E, Khanlou H, et al. 2008. Efficacy and safety of oncedaily darunavir/ritonavir versus lopinavir/ritonavir in treatment-naive HIV-1-infected patients at week 48. Aids, 22:1389-97.

Panel on Antiretroviral Guidelines for Adults and Adolescents Guidelines for the use of antiretroviral agents in HIV-1-infected adults and adolescents. Department of Health and Human Services January 29, 2008;1-128. URL: http://www.aidsinfo.nih.gov/ContentFiles/ AdultandAdolescentGL.pdf.

Rodriguez-French A, Boghossian J, Gray GE, et al. 2004. The NEAT study: a 48-week open-label study to compare the antiviral efficacy and safety of GW433908 versus nelfinavir in antiretroviral therapy-naive HIV-1-infected patients. J Acquir Immune Defic Syndr, 35:22-32.
Ruane,PJ, Luber AD, Wire MB, et al. 2007. Plasma amprenavir pharmacokinetics and tolerability following administration of 1,400 milligrams of fosamprenavir once daily in combination with either 100 or 200 milligrams of ritonavir in healthy volunteers. Antimicrob Agents Chemother, 51:560-5.

Sadler BM, Hanson CD, Chittick GE, et al. 1999. Safety and pharmacokinetics of amprenavir (141W94), a human immunodeficiency virus (HIV) type 1 protease inhibitor, following oral administration of single doses to HIV-infected adults. Antimicrob Agents Chemother, 43:1686-92.

Sadler BM, Piliero PJ, Preston SL, et al. 2001. Pharmacokinetics and safety of amprenavir and ritonavir following multiple-dose, co-administration to healthy volunteers. Aids, 15:1009-18.

Smith KY, Weinberg WG, Dejesus E, et al. 2008. Fosamprenavir or atazanavir once daily boosted with ritonavir $100 \mathrm{mg}$, plus tenofovir/ emtricitabine, for the initial treatment of HIV infection: 48-week results of ALERT. AIDS Res Ther, 5:5.

Walmsley SR, Slim K, Ward J, et al. 2007. Saquinavir/r (SQV/r) BiD versus lopinavir/r (LPV/r) BiD, plus emtricitabine/tenofovir (FTC/TDF) QD as initial therapy in HIV-1 infected patients: the GEMINI study. 11 th European AIDS Conference. Madrid, Spain.

Wood R, Arasteh K, Stellbrink HJ, et al. 2004. Six-week randomized controlled trial to compare the tolerabilities, pharmacokinetics, and antiviral activities of GW433908 and amprenavir in human immunodeficiency virus type 1-infected patients. Antimicrob Agents Chemother, 48:116-23. 